\title{
NOMENCLATURE
}

\section{Issues on the Surface Integrity of Case Hardened Steel Materials}

\begin{tabular}{|c|c|c|}
\hline A & $=$ & Furnace Temperature in degree Celsius \\
\hline AISI & $=$ & American Iron and Steel Institute \\
\hline ANOVA & $=$ & Analysis of Variance \\
\hline $\mathrm{B}$ & $=$ & Quenching time in minutes \\
\hline $\mathrm{C}$ & $=$ & Tempering temperature in degree Celsius \\
\hline $\mathrm{CA}_{\mathrm{opt}}$ & $=$ & Case depth value obtained at optimum process variable A \\
\hline $\mathrm{CB}_{\mathrm{opt}}$ & $=$ & Case depth value obtained at optimum process variable B \\
\hline $\mathrm{CC}_{\mathrm{opt}}$ & $=$ & Case depth value obtained at optimum process variable $\mathrm{C}$ \\
\hline $\mathrm{CD}_{\mathrm{opt}}$ & $=$ & Case depth value obtained at optimum process variable D \\
\hline $\mathrm{CE}_{\mathrm{opt}}$ & $=$ & Case depth value obtained at optimum process variable $\mathrm{E}$ \\
\hline C.F & $=$ & Correction factor \\
\hline $\mathrm{D}$ & $=$ & Tempering time in minutes \\
\hline $\mathrm{E}$ & $=$ & Preheating temperature in degree Celsius \\
\hline $\mathrm{EN}$ & $=$ & European Standards published by the European Committee for Standardization \\
\hline fm & $=$ & Volume fraction of martensite \\
\hline G1 & $=$ & Group 1 \\
\hline $\mathrm{G} 2$ & $=$ & Group 2 \\
\hline $\mathrm{HA}_{\mathrm{opt}}$ & $=$ & Hardness value obtained at optimum process variable A \\
\hline $\mathrm{HB}_{\mathrm{opt}}$ & $=$ & Hardness value obtained at optimum process variable B \\
\hline $\mathrm{HC}_{\mathrm{opt}}$ & $=$ & Hardness value obtained at optimum process variable $\mathrm{C}$ \\
\hline $\mathrm{HD}_{\mathrm{opt}}$ & $=$ & Hardness value obtained at optimum process variable D \\
\hline $\mathrm{HE}_{\mathrm{opt}}$ & $=$ & Hardness value obtained at optimum process variable E \\
\hline HRA & $=$ & Rockwell Hardness A scale \\
\hline $\mathrm{HRC}$ & $=$ & Rockwell Hardness C scale \\
\hline $\mathrm{LA}_{\mathrm{opt}}$ & $=$ & Helix variation (Left) value obtained at optimum process variable A \\
\hline $\mathrm{LB}_{\mathrm{opt}}$ & $=$ & Helix variation (Left) value obtained at optimum process variable B \\
\hline $\mathrm{LC}_{\mathrm{opt}}$ & $=$ & Helix variation (Left) value obtained at optimum process variable $\mathrm{C}$ \\
\hline $\mathrm{LD}_{\mathrm{opt}}$ & $=$ & Helix variation (Left) value obtained at optimum process variable D \\
\hline $\mathrm{LE}_{\mathrm{opt}}$ & $=$ & Helix variation (Left) value obtained at optimum process variable E \\
\hline Ms & $=$ & Starting temperature of marteniste formation \\
\hline $\mathrm{M}_{\mathrm{f}}$ & $=$ & Temperature of martensite transformation ends \\
\hline $\mathrm{N}$ & $=$ & Number of levels \\
\hline $\mathrm{P}$ & $=$ & Power potential in $\mathrm{kW} /$ inch $^{2}$ \\
\hline Q & $=$ & Quench flow rate litres/minutes \\
\hline $\mathrm{r}$ & $=$ & Number of replicates \\
\hline $\mathrm{RA}_{\mathrm{opt}}$ & $=$ & Runout value obtained at optimum process variable A \\
\hline
\end{tabular}




\begin{tabular}{|c|c|c|}
\hline $\mathrm{RB}_{\mathrm{opt}}$ & $=$ & Runout value obtained at optimum process variable B \\
\hline $\mathrm{RC}_{\mathrm{opt}}$ & $=$ & Runout value obtained at optimum process variable $\mathrm{C}$ \\
\hline $\mathrm{RD}_{\mathrm{opt}}$ & $=$ & Runout value obtained at optimum process variable D \\
\hline $\mathrm{RE}_{\text {opt }}$ & $=$ & Runout value obtained at optimum process variable E \\
\hline $\mathrm{S}$ & $=$ & Scan speed in meter/minutes \\
\hline SSE & $=$ & Sum of Squares of Error \\
\hline SST & $=$ & Total sum of squares \\
\hline $\mathrm{SST}_{\mathrm{r}}$ & $=$ & Sum of Squares of Treatment with replicates \\
\hline SSA & $=$ & Sum of Squares of Variables \\
\hline $\mathrm{Tq}$ & $=$ & Quenchant temperature at which fraction of martensite is formed \\
\hline TTT & $=$ & Temperature-Time-Transformation diagram \\
\hline VHN & $=$ & Vickers Hardness Number \\
\hline$\beta$ & $=$ & Predicted mean response \\
\hline
\end{tabular}

\author{
N. Alagumurthi \\ (Guest Editor) \\ Department of Mechanical Engineering \\ Pondicherry Engineering College \\ Pondicherry-605014, \\ India \\ E-mail: alagumurthi@pec.edu
}

